\title{
Warm tachyonic inflation in warped background
}

\author{
Atri Deshamukhya ${ }^{1}$ and Sudhakar Panda ${ }^{2}$ \\ 1 Department of Physics,Assam University, Silchar-788011,Indid F $^{2}$ and \\ 2 Harish-Chandra Research Institute, Allahabad- 211019, Indid
}

\begin{abstract}
We analyze warm tachyonic inflation, proposed in the literature, but from the viewpoint of four dimensional effective action for tachyon field on a non-BPS D3-brane. We find that consistency with observational data on density perturbation and validity of effective action requires warped compactification. The number of background branes which source the flux is found to be of the order of 10 in contrast to the order of $10^{14}$ in the standard cold inflationary scenario.
\end{abstract}

\section{INTRODUCTION}

The inflationary mechanism [1, 2] though provides natural solutions to 'problems' viz. horizon, flatness etc of Standard Big Bang model - can't naturally generate the correct magnitude of initial density perturbations demanded from experimental observations [3]. Standard inflationary scenario is divided into two regimes - slow roll expansion and reheating phase which happen in two different time periods. It is assumed that exponential expansion places the universe in a super-cooled phase and subsequently thereafter the universe is reheated. In mid 90s it was recognized by Berera and Fang [4] that meshing these two isolated stages may resolve the disparities created by each separately. In Ref.[4], it was shown that slow-roll inflation [5] is parametrically consistent with a thermal component.

The resolution of the horizon problem, which underlies inflationary cosmology, is that at a very early time, the equation of state that dictates the expansion rate of the Universe was dominated by a vacuum energy density $\rho_{v}$, so that a small causally connected patch grew to a size that encompasses the comoving volume which becomes the observed universe today. In the standard (isentropic) inflationary scenarios, the radiation energy density $\rho_{\gamma}$ becomes negligible rapidly since it scales inversely with the fourth power of the scale factor. In such case, a short time reheating period terminates the inflationary period initiating the radiation dominated epoch.

But from general theory of relativity for inflation, $\rho_{\gamma}$ need not be negligible and the condition which needs to be satisfied is that $\rho_{\gamma}<\rho_{v}$. Inflation in the presence of non-negligible radiation is thus characterized by a non-isentropic expansion [6] and thermal seeds of density perturbations [4]. This can be realized in the warm inflation scenarios [7] where there is no need for reheating but it is possible to have a smooth transition to the radiation dominated epoch.

The basic idea of warm inflation is that the inflaton field is coupled to several other fields. As the inflaton relaxes toward its minimum energy configuration, it decays into lighter fields, generating an effective viscosity [8]. If this viscosity is large enough, the inflaton will reach a slow-roll regime, where its dynamics becomes over-damped. This overdamped regime has been analyzed in Ref.[9]. As one expects, over-damping is most successful for the case where the inflaton is coupled to a large number of fields which are thermally excited, i.e., have small masses compared to the ambient temperature of radiation. This result has important consequences for cosmological applications since overdamping must be efficient to get the correct number of e-folds before the end of inflation.

There seems to be other reasons which makes warm inflationary scenario more compelling. First, in this scenario, since the macroscopic dynamics of the background field and fluctuations [4, 10] are classical from the onset, there is no quantum-classical transition problem. Thus it overcomes a conceptual barrier that the supercooled picture could not get away with. Second, in warm inflation models, in regimes relevant to observation, the mass of the inflaton field is typically much larger than the Hubble scale. These models thus do not suffer from what is sometimes called the 'eta problem'. Finally, accounting for dissipative effects may be important in alleviating the initial condition problem of inflation [11].

The inclusion of thermal effects acts similar to a mass term which breaks the scale-symmetry of the zero-temperature theory [12]. In Ref. [13] it has been argued that the coupling constant fine-tuning problem is closely associated to this scale symmetry and breaking this scale symmetry can avoid this problem. We remark in passing that there has been debate in the literature 14] questioning the very possibility of warm inflation. In this note, we donot attempt to assert on this issue either way. On the other hand, assuming that it is possible, we argue that just like the cold inflationary scenario [15], even the warm inflation model needs warp compactification for the validity of low energy

*Electronic address: atri.deshamukhya@gmail.com

${ }^{\dagger}$ Electronic address: panda@mri.ernet.in 
effective action obtained from ten dimensional string theory.

We organize the paper as follows: in next section we briefly review the tachyon warm inflationary model put forward by Herrera et al [16]. In section III, we discuss the low energy tachyon effective action and the constraints on parameters coming from compactification. In section IV, we analyze the model including the effect of radiation and discuss the consequences of warm inflation. The last section is devoted to conclusion of our analysis.

\section{WARM TACHYON INFLATION}

In this section we review the model of warm tachyonic inflation proposed by Herrera et al .

The authors analyzed FRW cosmological model in terms of an effective fluid with energy density, $\rho_{T}$ and pressure, $p_{T}$ associated with the tachyon field $T$ and defined respectively by

$$
\rho_{T}=\frac{V(T)}{\sqrt{1-\dot{T}^{2}}}, \quad p_{T}=-V(T) \sqrt{1-\dot{T}^{2}}
$$

where $V(T)$ is the effective potential associated with the tachyon field. The dynamics of the cosmological model, in the warm inflationary scenario is governed by the equations:

$$
\begin{gathered}
H^{2}=\frac{1}{3 M_{P}^{2}}\left(\rho_{T}+\rho_{\gamma}\right) \\
\dot{\rho}_{T}+3 H\left(\rho_{T}+p_{T}\right)=-\Gamma \dot{T}^{2} \\
\Longrightarrow \frac{\ddot{T}}{\left(1-\dot{T}^{2}\right)}+3 H \dot{T}\left(1+\frac{\Gamma \sqrt{1-\dot{T}^{2}}}{3 H V}\right)+\frac{V_{, T}}{V}=0
\end{gathered}
$$

and

$$
\dot{\rho}_{\gamma}+4 H \rho_{\gamma}=\Gamma \dot{T}^{2}
$$

where $H=\dot{a} / a$ is the Hubble factor, $a$ is the scale factor, $\rho_{\gamma}$ is the energy density of the radiation field and $\Gamma$ is the dissipation coefficient which is responsible for the decay of the tachyon field into radiation during the inflationary epoch. The presence of the energy density of radiation and the dissipation coefficient distinguishes the warm inflation from the cold inflation dynamics.

The authors used the potential that corresponds to the tachyon field living on a D-brane of the bosonic string theory, namely $V(t)=V_{0} \exp [-\alpha T]$ and derived the constraints on the $V_{0}$ and $\alpha$ by solving the above set of equations and imposing observational constraints on cosmological parameters coming from the density perturbation theory (see [16] for details). However, in such an approach the constraints coming from string theory, namely the validity of the low energy effective action (to be discussed below) as well as the height of the tachyon potential, which is fixed for a given brane in terms of its tension, did not play any role. Thus their analysis is phenomenologically motivated rather than describing a viable inflationary (warm) model which is derived from compactified string theory. On the contrary, these issues have been discussed earlier in the cold inflationary scenario and we briefly review it in the next section.

\section{TACHYON EFFECTIVE ACTION AND COUPLING TO GRAVITY:}

The effective action for the tachyon field, living on a non-BPS D3-brane, when coupled to four dimensional gravity can be written as [17]

$$
S=\int d^{4} x \sqrt{-g}\left(\frac{M_{P}^{2}}{2} R-A V(T) \sqrt{1+B g^{\mu \nu} \partial_{\mu} T \partial_{\nu} T}\right)
$$


where $V(T)$ is the positive definite tachyon potential which has a maximum at $T=0$ with normalization $V(0)=1$ and $V \rightarrow 0$ as $T \rightarrow \infty$. $A$ and $B$ are dimensionful constants which depends on string length and the closed string coupling constant $g$. Note that the tachyon field here is dimensionless.

In the conventional dimensional reduction approach $A, B$ and $M_{P}^{2}$ are given by

$$
\begin{aligned}
A & =\frac{\sqrt{2}}{(2 \pi)^{3} g \alpha^{\prime 2}} \\
B & =8 \ln 2 \alpha^{\prime} \\
M_{P}^{2} & =\frac{v}{g^{2} \alpha^{\prime}}
\end{aligned}
$$

corresponding to the case of space-filling non-BPS D3-brane in type IIA theory. $v$ is related to the volume $V_{6}$ of the compact manifold as

$$
v=\frac{2 V_{6}}{(2 \pi)^{7} \alpha^{\prime 3}}
$$

and is a dimensionless constant. Note that the tension of the non-BPS brane, which usually comes with the tachyon potential, has been included in $A$ and we will consider the potential function $V(T)$ to be $\exp \left[-T^{2}\right]$. This is motivated from string field theory and gives a good description for small $T$ which is assumed to be accurate for the inflationary epoch. As mentioned, for the validity of the above effective action one requires $g \ll 1$ and $v \gg 1$.

The energy density $\rho_{T}$ and pressure $p_{T}$ for the tachyon field derived from the above action are given as:

$$
\begin{aligned}
\rho_{T} & =\frac{A V(T)}{\sqrt{1-B \dot{T}^{2}}} \\
p_{T} & =-A V(T) \sqrt{1-B \dot{T}^{2}} .
\end{aligned}
$$

The inflation dynamics is governed by the Einstein equations for FRW background metric and the tachyon field equation derived from the above action [18] (see also [19]). These dynamical equations involve the pressure density and energy density as defined in the above and hence depend upon $A$ and $B$. Solving these equations and demanding cosmological parameters to be consistent with observational data put severe constraints on $A$ and $B$. In particular, it was observed in [20] that in this formalism, it is difficult to meet the second slow roll condition ( $\eta$-problem), besides the problem of ensuring the validity of low energy limit of string compactification; namely $g \ll 1$ and $v \gg 1$. The solutions to these problems were found in Ref [15] by considering a compactification to produce an warped background where the definition of $A$ and $B$ changes and will be defined in a later section. It was assumed that all the moduli fields are stabilized and the warped background was created by a stack of D6-branes. However, to ensure that the cosmological parameters to be consistent with the observational data, it was found that the number of required background D6branes is $10^{14}$ which seems to be too high. Thus it is natural to re-examine this issue in the warm inflationary scenario and we carry out this analysis in the next sction. Our interesting observation is that the minimum number of required background branes is less than ten.

\section{WARM INFLATION IN NON-WARPED AND WARPED BACKGROUND:}

As mentioned in section II, in the warm inflationary scenario, i.e when thermal effects are taken into account, the corresponding equations are modified due to the contribution of radiation energy density. For the effective action (5) and corresponding energy density and pressure density in (10), the inflation dynamics is governed by the following equations :

$$
\begin{gathered}
H^{2}=\frac{1}{3 M_{P}^{2}}\left(\rho_{T}+\rho_{\gamma}\right) \\
\dot{\rho}_{T}+3 H\left(\rho_{T}+p_{T}\right)=-\Gamma \dot{T}^{2} \\
\Longrightarrow \frac{\ddot{T}}{\left(1-B \dot{T}^{2}\right)}+3 H \dot{T}\left(1+\frac{\Gamma \sqrt{1-B \dot{T}^{2}}}{3 H A B V}\right)+\frac{V_{T}}{B V}=0
\end{gathered}
$$


and

$$
\dot{\rho}_{\gamma}+4 H \rho_{\gamma}=\Gamma \dot{T}^{2}
$$

In our notation, overdots represent derivative with respect to time and ()$_{,_{T}} \equiv \frac{\partial}{\partial T}$.

In the inflationary epoch, it is assumed that

$$
\begin{aligned}
& \rho_{T} \sim A V \\
& \rho_{T}>\rho_{\gamma} .
\end{aligned}
$$

Since in the slow-roll regime $\dot{T}^{2} \ll 1$ and $\ddot{T} \ll\left(3 H+\frac{\Gamma}{A B V}\right) \dot{T}$ and defining the dissipation rate $r$ as

$$
r \equiv \frac{\Gamma}{3 H A B V}
$$

the Friedmann equation and the slow-roll equation of motion for the tachyon field respectively takes the form:

$$
\begin{aligned}
H^{2} & =\frac{A V}{3 M_{P}^{2}} \\
3 H B(1+r) \dot{T} & =-\frac{V, T}{V} .
\end{aligned}
$$

For a quasi-stable radiation process during inflationary epoch, we need

$$
\dot{\rho_{\gamma}} \ll 4 H \rho_{\gamma}
$$

and

$$
\dot{\rho_{\gamma}} \ll \Gamma \dot{T}^{2}
$$

Hence from Eq.(10), we obtain

$$
\begin{aligned}
\rho_{\gamma} & =\frac{\Gamma \dot{T}^{2}}{4 H} \\
& =\frac{r M_{P}^{2}}{4 B(1+r)^{2}}\left(\frac{V, T}{V}\right)^{2} \\
& =\sigma T_{\gamma}{ }^{4}
\end{aligned}
$$

where $T_{\gamma}$ is the radiation temperature and $\sigma$ is the Stephan-Boltzmann constant.

The standard slow-roll parameters can then be written as :

$$
\begin{aligned}
\varepsilon & \equiv-\frac{\dot{H}}{H^{2}}=\frac{M_{P}^{2}}{2 A B(1+r) V}\left(\frac{V, T}{V}\right)^{2} \\
\eta & \equiv-\frac{\ddot{H}}{H \dot{H}}=\frac{M_{P}^{2}}{A B(1+r) V}\left[\frac{V,_{T T}}{V}-\frac{1}{2}\left(\frac{V,_{T}}{V}\right)^{2}\right] .
\end{aligned}
$$

End of inflation is marked by $\varepsilon \simeq 1$ which also implies

$$
\rho_{T} \simeq 2 \frac{(1+r)}{r} \rho_{\gamma}
$$

Number of e-folds before the end of inflation is given by

$$
N(T)=-\frac{A B}{M_{P}^{2}} \int_{T}^{T_{e}} \frac{V^{2}}{V, T}(1+r) d T^{\prime} .
$$

Using perturbed FRW metric in the longitudinal gauge and following the procedure depicted in Ref. [16] one can express the fluctuation of tachyon field as

$$
\delta T=C(\ln V), T \exp (\mathfrak{F}(T))
$$


where $C$ is a constant and $\mathfrak{F}$ is given by:

$\mathfrak{F}(T)=-\int\left[\frac{1}{3 H+\Gamma / A B V}\left(\frac{\Gamma}{A B V}\right)_{,_{T}}+\frac{9}{8} \frac{(2 H+\Gamma / A B V)}{(3 H+\Gamma / A B V)^{2}} \frac{(\ln V)_{, T}}{A B V}\left(\Gamma+4 H A B V-\frac{\Gamma,_{T}(\ln V),_{T}}{12 H B(3 H+\Gamma / A B V)}\right)\right] d T$.

The density perturbation is defined to be [16]

$$
\delta_{H}=\frac{2}{5} \frac{\exp [-\mathfrak{F}(T)]}{(\ln V),_{T}} \delta T .
$$

If one restricts to the region of high dissipation where the dissipation parameter $\Gamma$ is much higher than the product of expansion rate of the universe and the potential energy of the scalar field i.e $\Gamma \gg 3 H A B V$ then using Eq.(21) in $r \gg 1$ limit one can write

$$
\delta_{H}^{2}=\frac{4}{225} \frac{\exp [-2 \tilde{\mathfrak{F}}(T)]}{(H B r \dot{T})^{2}}(\delta T)^{2}
$$

where

$$
\tilde{\mathfrak{F}}(T)=-\int\left[\frac{1}{3 H r}\left(\frac{\Gamma}{A B V}\right)_{,_{T}}+\frac{9}{8}(\ln V)_{,_{T}}\left(1-\frac{\ln \Gamma,_{T}(\ln V)_{, T}}{36 H^{2} B r}\right)\right] d T .
$$

In warm inflationary scenario, the fluctuations of the tachyon field are generated by thermal interaction with the radiation field and in the high dissipation regime, following Ref. [21] this can be written as:

$$
(\delta T)^{2} \simeq \frac{k_{F} T_{\gamma}}{2 \pi^{2} M_{P}^{2}}
$$

Here $k_{F}$ corresponds to the freeze-out scale at which thermally excited fluctuations are damped by dissipation. This freeze-out wave number is defined as $k_{F}=H \sqrt{3 r} \geq H$.

Using equations (19), (27) and (29) we find

$$
\delta_{H}^{2} \approx \frac{1}{25 \sqrt{3} \pi^{2}} \exp [-2 \mathfrak{F}(T)]\left[\left(\frac{1}{\tilde{\varepsilon}}\right)^{3} \frac{9}{2 r^{2} \sigma M_{P}^{4} A B^{4} V}\right]^{1 / 4}
$$

where $\tilde{\varepsilon}$ is the slow-roll parameter in the $r \gg 1$ limit.

Note that in the high dissipation regime the slow-roll parameters given by equations (20) and (21) take the forms-

$$
\begin{aligned}
\tilde{\varepsilon} & =\frac{M_{P}^{2}}{2 r A B V}\left[\frac{V,_{T}}{V}\right]^{2} \\
\tilde{\eta} & =\frac{M_{P}^{2}}{r A B V}\left[\frac{V,_{T T}}{V}-\frac{1}{2}\left(\frac{V,_{T}}{V}\right)^{2}\right]
\end{aligned}
$$

The scalar spectral index is given by:

$$
\begin{aligned}
n_{s}-1 & =\frac{d \ln \delta_{H}^{2}}{d \ln k} \\
& =\frac{3 \tilde{\eta}}{2}+\tilde{\varepsilon}\left[\frac{2 V}{V, T}\left(2 \tilde{\mathfrak{F}}(T),_{T}-\frac{r, T}{4 r}\right)-\frac{5}{2}\right]
\end{aligned}
$$

and hence the running of the spectral index is

$$
\begin{aligned}
& \alpha_{s}=\frac{d n_{s}}{d \ln k} \\
& \left.=-\frac{2 V \tilde{\epsilon}}{V, T}\left\{\frac{3 \tilde{\eta}_{, T}}{2}+\frac{\tilde{\epsilon}, T}{\tilde{\epsilon}}\left(n_{s}-1-\frac{3 \tilde{\eta}, T}{2}\right)+2 \tilde{\epsilon}\left[\left(\frac{V}{V, T}\right),_{T}\left(2 \tilde{\mathfrak{F}}_{T}-\frac{(\ln r)_{T}}{4}\right)+\left(\frac{V}{V, T}\right)\left(2 \tilde{\mathfrak{F}}_{,_{T}}-\frac{(\ln r), T T}{4}\right)\right]\right\} 37\right)
\end{aligned}
$$


Power spectrum of tensor modes in this scenario has an extra temperature dependence factor coth $\left[\frac{k}{2 T}\right]$ (see Ref.[22] ) and is given by:

$$
A_{g}^{2}=\frac{H^{2}}{2 \pi^{2} M_{P}^{2}} \operatorname{coth}\left[\frac{k}{2 T_{\gamma}}\right]
$$

Thus the tensor spectral index is

$$
\begin{aligned}
n_{T} & =\frac{d}{d \ln k} \ln \left[\frac{A_{g}^{2}}{\operatorname{coth}\left[k / 2 T_{\gamma}\right]}\right] \\
& =-2 \varepsilon .
\end{aligned}
$$

Power spectrum of the scalar modes on the other hand is given by

$$
\begin{aligned}
P_{\mathcal{R}} & =\frac{25}{4} \delta_{H}^{2} \\
& =\frac{1}{2 \sqrt{2}}\left[\frac{A^{2}}{M_{P}^{10} B} r \frac{V^{8}}{V,,_{T}^{6}}\right]^{1 / 4} \exp [-2 \tilde{\mathfrak{F}}] .
\end{aligned}
$$

Hence tensor to scalar ratio takes the form

$$
\begin{aligned}
R\left(k_{0}\right) & =\left.\frac{A_{g}^{2}}{P_{\mathcal{R}}}\right|_{k=k_{0}} \\
& =\left.\frac{\sqrt{2} \sigma^{2}}{3}\left[\frac{A^{2} B}{M_{P}^{6} r} \frac{V^{12}}{V_{, T}^{6}}\right]^{1 / 4} \exp [2 \tilde{\mathfrak{F}}] \operatorname{coth}\left[\frac{k}{2 T_{\gamma}}\right]\right|_{k=k_{0}} .
\end{aligned}
$$

\section{A. Numerical Analysis}

As motivated earlier, we now work with the explicit potential function $V(T)=e^{-T^{2}}$. The dissipation coefficient $\Gamma$ can de related to the radiation temperature using Eq.(19).And we have

$$
\tilde{\mathfrak{F}}(T)=-2 \frac{V^{\prime \prime}}{V^{\prime}}\left(1-\frac{3}{8} \frac{\sigma T_{\gamma}^{4}}{A V}\right)+\frac{3}{8} \frac{V^{\prime}}{V}\left(1-\frac{\sigma T_{\gamma}^{4}}{2 A} \frac{V^{\prime}}{V}\right) .
$$

Similarly, using Eq.(19), one can also express the slow roll parameters in terms of radiation temperature as follows:

$$
\begin{aligned}
& \tilde{\varepsilon}=2 \sigma \frac{T_{\gamma}^{4}}{A} \frac{1}{V} \\
& \tilde{\eta}=4 \sigma \frac{T_{\gamma}^{4}}{A}\left[\frac{V, T T}{V_{T}^{2}}-\frac{1}{2 V}\right] .
\end{aligned}
$$

In terms of these parameters, the cosmological observable can be expressed as

$$
\begin{aligned}
n_{s} & =1+\frac{3}{2} \tilde{\eta}+\tilde{\varepsilon}\left[-\frac{3 \sigma T_{\gamma}^{4}}{4 A} \frac{V^{\prime}}{V}-\frac{3\left(\sigma T_{\gamma}^{4} / A-3 V\right) V,_{T T}}{V,_{T}^{2}}\right] \\
n_{T} & =-\tilde{\varepsilon} \\
\alpha_{s} & =-\frac{2 V \tilde{\varepsilon}}{V, T}\left[\frac{3 \tilde{\eta},_{T}}{2}+\tilde{\varepsilon}_{T}\left(-\frac{3 \sigma T_{\gamma}^{4}}{4 A} \frac{V^{\prime}}{V}-\frac{3\left(\sigma T_{\gamma}^{4} / A-3 V\right) V,_{T T}}{V,_{T}^{2}}\right)\right] \\
& -\frac{4 V \tilde{\varepsilon}^{2}}{V, T}\left[\left(\frac{V}{V_{,}}\right),_{T}\left(2 \tilde{\mathfrak{F}}_{T}-\frac{(\ln r)_{T}}{4}\right)+\frac{V}{V,,_{T}}\left(2 \tilde{\mathfrak{F}}_{T T}-\frac{(\ln r),_{T T}}{4}\right)\right] \\
P_{\mathcal{R}} & =\frac{1}{4 \pi^{2} \sigma^{2}}\left[\frac{A^{2}}{M_{P}^{8} B^{2} T_{\gamma}^{4}} r \frac{V^{6}}{V_{, T}^{4}}\right]^{1 / 4} \exp [-2 \tilde{\mathfrak{F}}] \\
R\left(k_{0}\right) & =\left.\frac{2 \sigma^{2}}{3}\left[T_{\gamma}^{4} \frac{A^{2} B^{2}}{M_{P}^{8}} \frac{V_{T}^{4}}{V^{2}}\right]^{1 / 4} \exp [2 \tilde{\mathfrak{F}}] \operatorname{coth}\left[\frac{k}{2 T_{\gamma}}\right]\right|_{k=k_{0} .} .
\end{aligned}
$$


All these observable quantities are to be evaluated at $T_{*}$, which is the value of the tachyon field at roughly 60 e-folds before the end of inflation. We consider the following strategy for our numerical estimation of $A$ and $B$ which will lead to the analysis of constraints on $g$ and $v$ :

1. From the condition for end of inflation, $\tilde{\varepsilon}\left(T_{e}\right)=1, T_{e}$ is obtained as a function of $T_{\gamma}^{4} / A$.

2. Fixing $N_{e}$ to be $60, T_{*}$ is evaluated as a function of $T_{e}$.

3. The lower limit for $T_{\gamma}^{4} / A$ is found out for which the upper bound on running of spectral index is consistent with the observational data whereas the upper limit is fixed from the condition that minimum value of $N_{e}$ is 60 .

4. Using WMAP data [3] for $P_{\mathcal{R}}$ i.e. $P_{\mathcal{R}} \leq 2.3 \times 10^{-9}$, range of $A^{2} /\left(T_{\gamma}^{4} M_{P}^{8} B^{2}\right)$ is obtained.

We quote below the range for $A / M_{P}^{4}, \sqrt{A / M_{P}^{6} B}$ obtained from our above numerical estimation.

$$
\begin{gathered}
10^{-9} \leq \frac{A}{M_{P}^{4}}<10^{-3} \\
2.5 \leq \sqrt{\frac{A}{M_{P}^{6} B}} \leq 10^{32}
\end{gathered}
$$

In this analysis we have used $k_{0}=0.002 \mathrm{Mpc}^{-1}$ and $T_{\gamma}=0.24 \times 10^{16} \mathrm{GeV}[16] . \sigma$ is taken to be of the order of unity. We have confirmed that the slow roll conditions are satisfied for these values of $A$ and $T_{\gamma}$.

Using the above we predict the bounds on the rest of the observable as below : The spectral index is in the range

$$
0.9480 \leq n_{s} \leq 0.9636
$$

Running of the spectral index is found to be negative and lie in the range

$$
-0.00093 \leq \alpha_{s} \leq-0.00099
$$

The tensor-scalar ratio is found to be negligible over the whole range of parameters. These are well within the limit given by WMAP 5years data [3].

We now proceed to analyse the above results in terms of the string theory parameters $g$ and $v$. For each corresponding set of values of $A / M_{P}^{4}$ and $A^{2} / M_{P}^{12} B^{2}$ we can solve for $g$ and $v$ as :

$$
\begin{aligned}
g^{7} & =\frac{2^{13 / 2} \ln 2}{(2 \pi)^{3}} \frac{A^{2} / M_{P}^{12} B^{2}}{\left(A / M_{P}^{4}\right)^{3}} \\
v^{14} & =\frac{2^{10} \ln 2}{(2 \pi)^{2} 4} \frac{A 2 / M_{p}^{12} B^{2}}{\left(A / M_{P}^{4}\right)^{10}} .
\end{aligned}
$$

We tabulate below the values of $g$ and $v$ corresponding to the range of $A / M_{P}^{4}$ and $A^{2} /\left(M_{P}^{12} B^{2}\right)$ obtained earlier.

\begin{tabular}{|c|c|c|c|}
\hline$A / M_{P}^{4}$ & $\sqrt{\frac{A}{M_{P}^{6} B}}$ & $g$ & $v$ \\
\hline $10^{-9}$ & 2.48 & $9.9 \times 10^{3}$ & $2.38 \times 10^{5}$ \\
\hline $10^{-7}$ & $7.85 \times 10^{10}$ & $1.38 \times 10^{9}$ & $8.87 \times 10^{6}$ \\
\hline $10^{-5}$ & $4.28 \times 10^{21}$ & $1.38 \times 10^{21}$ & $3.86 \times 10^{8}$ \\
\hline $10^{-3}$ & $7.84 \times 10^{31}$ & $2.67 \times 10^{19}$ & $1.23 \times 10^{10}$ \\
\hline
\end{tabular}

Table I

From this table it is clear that in the allowed parameter range for $A$ and $B$ for which the cosmological observables are consistent with experimental data, the required constraint $g \ll 1$ and $v \gg 1$ is never satisfied simultaneously.

\section{B. Warm Inflation in Warped background}

As concluded in the previous section, within conventional compactification it is not possible to obtain physical parameters of inflation consistent with observations keeping $g \ll 1$ and $v \gg 1$. To solve this problem, we now redo our analysis considering the warped compactification [23] similar to the analysis of [15] for the case of cold inflation. 
We introduce warping by considering the ten-dimensional string frame metric of the form

$$
d s^{2}=e^{2 C(y)} g_{\mu \nu}(x) d x^{\mu} d x^{\nu}+g_{m n}(y) d y^{m} d y^{n}
$$

where $e^{2 C(y)}$ being the warp factor which can take very small values. Here $x$ and $y$ denote the coordinates on the four-dimensional non-compact and sis-dimensional compact space respectively. In addition, the dilaton field is allowed to vary over the compact manifold as

$$
\phi=\phi_{0}+\phi(y)
$$

Such warping can be produced by introducing a number of background D6-branes and an appropriate number of O6-planes for charge conservation.

In this background, clearly the definitions of $A$ and $B$ gets modified as:

$$
\begin{aligned}
A & =\frac{\sqrt{2} e^{4 C-\phi}}{(2 \pi)^{3} g \alpha^{\prime 2}} \\
B & =8 \ln 2 \alpha^{\prime} e^{-2 C} .
\end{aligned}
$$

The functions $C$ and $\phi$ here are subject to the solutions of equations of motion derived from supergravity theory.

The four dimensional Planck mass in such a compactification is found to be

$$
M_{P}^{2}=\frac{\tilde{v}}{g^{2} \alpha^{\prime}}
$$

where $g=e^{\phi_{0}}$ and the warped volume $\tilde{v}$ is

$$
\tilde{v}=\frac{2}{(2 \pi)^{7} \alpha^{\prime 3}} \int d^{6} y \sqrt{g_{6}} e^{-2 \phi+2 C} .
$$

By choosing average value of $e^{-2 \phi+2 C}$ of the order of one, $\tilde{v}$ can be taken of same order as $v$.

Within the supergravity approximation one can derive the following expressions for the warp factor and the dilaton field:

$$
\begin{aligned}
e^{2 C} & =\left(g N_{\min }\right)^{-2 / 3} \\
e^{-\phi} & =g N_{\min }
\end{aligned}
$$

where $N_{\min }$ is the minimum number of background D6-branes. For slow-roll conditions to be satisfied one requires $e^{4 C-\phi} \gg 1$.

In such a background, the relation of $g$ and $v$ with parameters $A$ and $B$ depends on the warp factors and are given by:

$$
\begin{aligned}
g e^{\phi} & =\left(\frac{2^{13 / 2} \ln 2}{(2 \pi)^{3}} \frac{A^{2} / M_{P}^{12} B^{2}}{\left(A / M_{P}^{4}\right)^{3}}\right)^{\frac{1}{7}} \\
v e^{-2 C+\phi} & =\left(\frac{2^{10} \ln 2}{(2 \pi)^{24}} \frac{A 2 / M_{p}^{12} B^{2}}{\left(A / M_{P}^{4}\right)^{10}}\right)^{\frac{1}{14}}
\end{aligned}
$$

Eliminating $e^{\phi}$ and $e^{2 C}$ by the help of equations (62) and (63), the relation between $g$ and $v$ is found to be:

$$
g=2563 \frac{\left(A / M_{P}^{4}\right)^{12 / 7}}{\left(A^{2} / M_{P}^{12} B^{2}\right)^{1 / 14}} v^{3}
$$

Following our analysis in the previous section, we furnish the reinterpreted values of string coupling $g$ and volume of compact space $v$ in the light of warped background in table below.

We observe that it is possible to achieve, simultaneously, $g \ll 1$ and $v \gg 1$ for the whole range of papameters.

Using the equations (62) and (63) and the condition coming from slow-roll i.e $e^{4 C-\phi} \gg 1$, the minimum number of background D6- branes required to produce the required background is found to be less than ten. This is a significant improvement over the cold inflation model where the minimum number of such branes required was found to be $10^{14}$ [15]. 


\begin{tabular}{|c|c|c|c|c|}
\hline$A / M_{P}^{4}$ & $\sqrt{A / M_{P}^{6} B}$ & $g e^{\phi}$ & $v e^{-2 C+\phi}$ & $v$ for $g=.01$ \\
\hline $10^{-9}$ & 2.48 & $9.9 \times 10^{3}$ & $2.38 \times 10^{5}$ & 2363 \\
\hline $10^{-7}$ & $7.85 \times 10^{10}$ & $1.38 \times 10^{9}$ & $8.87 \times 10^{6}$ & 1716 \\
\hline $10^{-5}$ & $4.28 \times 10^{21}$ & $1.38 \times 10^{21}$ & $3.86 \times 10^{8}$ & 1301 \\
\hline $10^{-3}$ & $7.84 \times 10^{31}$ & $2.67 \times 10^{19}$ & $1.23 \times 10^{10}$ & 889 \\
\hline
\end{tabular}

Table II

\section{DISCUSSION}

In this work we investigated the viability of warm tachyonic inflationary scenario . Our analysis revealed that, though with a string theory motivated inflaton potential it is possible to get all the cosmological observables within the experimental bounds, it is not possible to comply with the validity of low energy effective action obtained from the conventional toroidal compactification namely the string coupling constant to be much less than unity and the volume of compact space to be larger than unity. On the contrary these problems could be resolved in an warped compactification. Moreover, an interesting observation is that the number of background D6-branes required to produce the necessary warped background turned out to be less than ten. This can be contrasted with the corresponding number $10^{14}$ in a cold inflation scenario. Our analysis assumed that all moduli fields are stabilized. We point out that it has been observed in 24] that it is impossible, in general flux compactification scheme, to cure the " $\eta$-problem" of inflation models derived from Type IIA string theory (the case we studied here) where any of the moduli fields play the role of an inflaton. Thus, in such a setup, it may be a welcome fact to trade the tachyon field as the inflaton. In principle, the introduction of a non-BPS brane can have some effect, through a change in Kähler potential, on the moduli stabilization, but we have not taken this into account in our present analysis. We hope to report on this in future. However, in the context of cold inflation, this issue is addressed partially in [25].

\section{Acknowledgements}

AD would like to thank the members, HRI, Allahabad for their continuous support to visit the Institute during which most of the work has been carried out.

[1] A. H. Guth, Phys. Rev. D 23 (1981) 347-352.

[2] A. J. Albrecht and P. J. Steinhardt, Phys. Rev. Lett. 48, 1220-1223 (1982). A.Linde, Particle Physics and inflationary cosmology (Gordon and Breach, New York, 1990).

[3] E. Komatsu et al. [WMAP Collaboration], Astrophys. J. Suppl. 180, 330-376(2009) arXiv:0803.0547 [astro-ph]].

[4] A. Berera and L. Z. Fang, Phys. Rev. Lett. 74, 1912-1915 (1995) arXiv:astro-ph/9501024.

[5] J. M. Bardeen, P. J. Steinhardt and M. S. Turner, Phys. Rev. D 28, 679 (1983).

A. D. Linde, Phys. Lett. B 129, 177-181 (1983).

[6] H. P. de Oliveira and R. O. Ramos, Phys. Rev. D 57, 741-749 (1998) arXiv:gr-qc/9710093.

E. Gunzig, R. Maartens and A. V. Nesteruk, Class. Quant. Grav. 15, 923-932 (1998) arXiv:astro-ph/9703137.

A. Berera, Phys. Rev. D 55, 3346-3357 (1997) arXiv:hep-ph/9612239.

[7] A. Berera,Phys. Rev. Lett. 75, 3218 (1995) arXiv:astro-ph/9509049.

A. Berera, Phys. Rev. D 54, 2519-2534 (1996) arXiv:hep-th/9601134.

[8] M. Gleiser and R. O. Ramos, Phys. Rev. D 50, 2441-2455 (1994) arXiv:hep-ph/9311278.

A. Hosoya and M. a. Sakagami, Phys. Rev. D 29, 2228 (1984).

M. Morikawa, Phys. Rev. D 33, 3607 (1986).

[9] A. Berera, M. Gleiser and R. O. Ramos, Phys. Rev. D 58, 123508 (1998) arXiv:hep-ph/9803394.

[10] A. Berera, Nucl. Phys. B 585, 666 -714(2000) arXiv:hep-ph/9904409.

[11] A. Berera and C. Gordon, Phys. Rev. D 63, 063505 (2001) arXiv:hep-ph/0010280].

R. O. Ramos, Phys. Rev. D 64, 123510 (2001) arXiv:astro-ph/0104379.

[12] A. Berera, Phys. Rev. Lett. 75, 3218 (1995) arXiv:astro-ph/9509049.

[13] B. Ratra, Phys. Lett. B 260, 21-26 (1991).

[14] J. Yokoyama and A. D. Linde, Phys. Rev. D 60, 083509 (1999) arXiv:hep-ph/9809409.

[15] J. Raeymaekers, JHEP 0410, 057 (2004) arXiv:hep-th/0406195.

P. Chingangbam, S. Panda and A. Deshamukhya, JHEP 0502, 052 (2005) arXiv:hep-th/0411210. 
[16] R. Herrera, S. del Campo and C. Campuzano, JCAP 0610, 009 (2006) arXiv:astro-ph/0610339.

[17] A. Sen, JHEP 9910, 008 (1999) arXiv:hep-th/9909062.

M. R. Garousi, Nucl. Phys. B 584, 284-299 (2000) arXiv:hep-th/0003122.

E. A. Bergshoeff, M. de Roo, T. C. de Wit, E. Eyras and S. Panda, JHEP 0005, 009 (2000) arXiv:hep-th/0003221]. J. Kluson, Phys. Rev. D 62, 126003 (2000) arXiv:hep-th/0004106.

[18] M. Fairbairn and M. H. G. Tytgat, Phys. Lett. B 546, 1-7 (2002) arXiv:hep-th/0204070.

D. Choudhury, D. Ghoshal, D. P. Jatkar and S. Panda, Phys. Lett. B 544, 231 (2002) arXiv:hep-th/0204204.

[19] A. Mazumdar, S. Panda and A. Perez-Lorenzana, Nucl. Phys. B 614, 101 (2001) arXiv:hep-ph/0107058.

[20] L. Kofman and A. Linde, JHEP 0207, 004 (2002) arXiv:hep-th/0205121].

[21] A. N. Taylor and A. Berera, Phys. Rev. D 62, 083517 (2000) arXiv:astro-ph/0006077].

[22] K. Bhattacharya, S. Mohanty and A. Nautiyal, Phys. Rev. Lett. 97, 251301 (2006) arXiv:astro-ph/0607049.

[23] C. S. Chan, P. L. Paul and H. L. Verlinde, Nucl. Phys. B 581, 156 (2000) arXiv:hep-th/0003236.

I. R. Klebanov and M. J. Strassler, JHEP 0008, 052 (2000) arXiv:hep-th/0007191.

S. B. Giddings, S. Kachru and J. Polchinski, Phys. Rev. D 66, 106006 (2002) arXiv:hep-th/0105097.

B. R. Greene, K. Schalm and G. Shiu, Nucl. Phys. B 584, 480-508 (2000) arXiv:hep-th/0004103.

[24] M. P. Hertzberg, S. Kachru, W. Taylor and M. Tegmark, JHEP 0712, 095 (2007) arXiv:0711.2512] [hep-th]].

[25] J. Ward, arXiv:0901.0080 [gr-qc]. 